\title{
One-Tap Wideband I/Q Compensation for Zero-IF Filters
}

\author{
Péter Kiss, Member, IEEE, and Vladimir Prodanov, Member, IEEE
}

\begin{abstract}
The I/Q imbalance is one of the performance bottlenecks in transceivers with stringent requirements imposed by applications such as 802.11a. The mismatch between the frequency responses of two analog low-pass filters, used, e.g., for channel selection in zero-IF receivers, makes this $I / Q$ imbalance frequency dependent. Usually, frequency-dependent $I / Q$ mismatch is estimated and corrected by adaptive techniques, which are complex to implement and may converge slowly due to noise. In this work, a simple, delay-based $\mathrm{I} / \mathrm{Q}$ compensation scheme is proposed based on an extensive statistical analysis. Its digital implementation uses only two coefficients, which are tuned by a one-step two-tone error estimation. Simulations show that this hardware-efficient scheme significantly reduces the $I / Q$ imbalance.
\end{abstract}

Index Terms-Complex filters, frequency-dependent I/Q compensation, I/Q filters, I/Q imbalance, sensitivity analysis, wideband I/Q calibration.

\section{I/Q MiSMATCH IN TRANSCEIVERS}

A simplified linear model of a zero-IF [1], [2] receiver $(\mathrm{RX})$ is shown in Fig. 1. Usually, the RX chain includes an antenna (A), a low-noise amplifier (LNA), a pair of mixers driven by quadrature local-oscillator (LO) signals I $\left(\mathrm{LO}_{1}\right)$ and $\mathrm{Q}\left(\mathrm{LO}_{2}\right)$, a pair of real low-pass filters (LPFs), a pair of analog-to-digital converters (ADCs) sampled at $f_{S}$, and a digital signal processor (DSP) [3]. An orthogonal frequency-division multiplexing (OFDM) DSP, used in 802.11a [4], inherently contains a fast-Fourier transform (FFT) block. A zero-IF transmitter (TX) chain starts with a DSP and is followed by a pair of digital-to-analog converters (DAC), a pair of LPFs, a mixer/LO block, a power amplifier (PA), and an antenna [3].

The main contributors of the RX or TX chain's I/Q imbalance $^{1}$ are the gain error $\left(\delta G_{1}\right)$ of the mixers, the phase error $\left(\delta P_{1}\right)$ in the LO signals, the gain $\left(\delta G_{2}(\omega)\right)$ and phase $\left(\delta P_{2}(\omega)\right)$ mismatch between the LPF's transfer functions, and, finally, the gain error $\left(\delta G_{3}\right)$ between the data converters (ADCs in RX, DACs in TX). The I/Q imbalance contribution of gain and phase errors can be modeled as a two-input two-output linear network with some inter-coupling coefficients [5]. These simple models can be individually applied to each block of mixers/LO, LPFs,

Manuscript received June 30, 2003; revised December 18, 2003. This paper was recommended by Associate Editor A. Wang.

The authors are with the Department of Communications Circuits Research, Agere Systems, Allentown, PA 18109 USA (e-mail: kpeter@agere.com; vprodanov@agere.com).

Digital Object Identifier 10.1109/TCSI.2004.829233

${ }^{1}$ Terminology used: I/Q mismatch or I/Q errors cause I/Q imbalance or I/Q leakage.

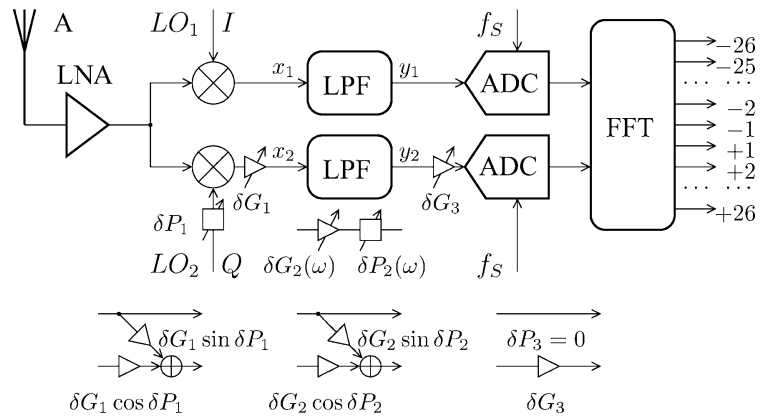

Fig. 1. I/Q leakage in an RX chain.

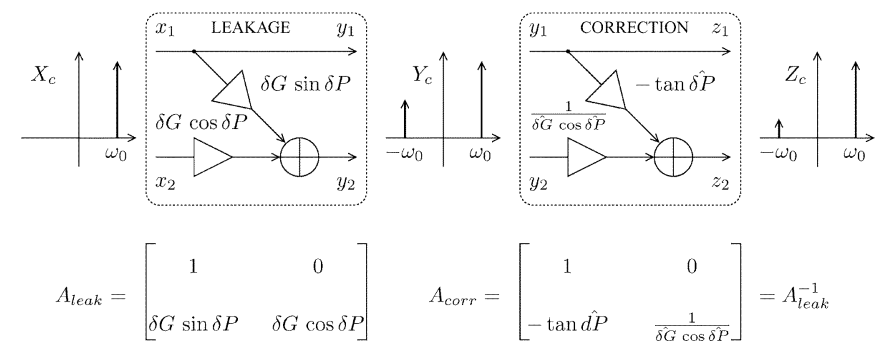

Fig. 2. Concept of I/Q leakage compensation.

and ADCs, as shown on the bottom of Fig. 1. In mathematical terms

$$
\vec{y}=A_{\text {leak }} \times \vec{x}
$$

where $\vec{y}=\left[y_{1}, y_{2}\right]^{\top}, \vec{x}=\left[x_{1}, x_{2}\right]^{\top}$, and $A_{\text {leak }}$ are given in Fig. 2. The resulting image rejection ratio (IMR) can be calculated by [5]

$$
\mathrm{IMR} \cong 10 \log _{10}\left|\frac{1+\delta G^{2}+2 \delta G \cos \delta P}{1+\delta G^{2}-2 \delta G \cos \delta P}\right| \quad \mathrm{dB} .
$$

A simple graphical derivation of (2) is presented in Appendix I. Detailed analytical calculations can be found in, e.g., [3], [6]-[8].

The concept of I/Q imbalance compensation is straightforward: whatever "leaks" due to I/Q mismatch can be cancelled by deliberately "leaking back" the same amount. To compensate for this I/Q leakage, first, the coefficients of the error matrix $A_{\text {leak }}$ should be estimated. Off-line estimation methods use one [5], [9] or multiple [10], [11] test tones and a measurement (FFT) block, or test-signal based adaptive tuning algorithms [12], [13]. Training signals are avoided in [8], [14], [15], which use blind, on-line adaptive methods to estimate and correct the I/Q imbalance. 
Once $A_{\text {leak }}$ was estimated, it is invertible since it has diagonal dominance. Therefore, a correction matrix $A_{\text {corr }}$ can be found by

$$
A_{\text {corr }}=A_{\text {leak }}^{-1}
$$

and used to cancel the I/Q leakage (Fig. 2). Since $A_{\text {corr }}$ should be tunable and requires high precision, it is usually implemented in the digital domain. Due to imperfect error estimation and finite word-length digital correction, some residual I/Q mismatch will affect the corrected output $Z_{c}$ (Fig. 2).

Note that the above-described I/Q imbalance compensation concept is valid for both RX and TX. While the RX uses digital "correction" or "compensation" ( $A_{\text {leak }}$ precedes $\left.A_{\text {corr }}\right)$, the TX uses digital "pre-distortion" ( $A_{\text {corr }}$ precedes $\left.A_{\text {leak }}\right)$.

The I/Q mismatch $\left(\delta G_{2}(\omega)\right.$ and $\left.\delta P_{2}(\omega)\right)$ of the filters is frequency dependent, while the I/Q mismatch of the front-end $\left(\delta G_{1}\right.$ and $\left.\delta P_{1}\right)$ and the $\operatorname{ADCs}\left(\delta G_{3}\right)$ can be considered frequency independent in first order. Therefore, the error matrix $A_{\text {leak }}(\omega)$ should be estimated for several frequencies. Thus, the implementation of the correction matrix $A_{\text {corr }}(\omega)$ becomes costly since it should be effective over the whole band of interest. Published frequency-dependent I/Q estimation/correction methods [10], [12], [14], [15] treat the zero-IF filters as a black box. However, its behavior can $a$ priori be predicted (presented in Section II) and used to considerably simplify the compensation hardware (presented in Section III). [5], [8], [9], [11], [13] deal with frequency-independent I/Q imbalance.

This paper is focused on zero-IF RX filters, but these concepts can be also extended for zero-IF TX filters by using the method proposed by, e.g., Burgin [16]. Also, $\delta G_{3}$ will be incorporated into $\delta G_{2}(\omega)$, and the subscripts of $\delta G_{2}$ and $\delta P_{2}$ will be ignored in the following sections for simplicity.

\section{I/Q MisMatch ANALYSIS}

\section{A. Two-Path Filtering}

In a conventional zero-IF architecture [1], [2], the pair of LPFs form a two-input two-output linear network with complex input $x_{c}(t) \doteq x_{1}(t)+j x_{2}(t)$ and complex output $y_{c}(t) \doteq$ $y_{1}(t)+j y_{2}(t)$ (Fig. 1). If the transfer functions of the upper and lower LPFs are defined as $H_{1}(\omega) \doteq Y_{1}(\omega) / X_{1}(\omega)=$ $B_{1}(\omega) / A_{1}(\omega)$ and $H_{2}(\omega) \doteq Y_{2}(\omega) / X_{2}(\omega)=B_{2}(\omega) / A_{2}(\omega)$, respectively, then

$$
\begin{aligned}
Y_{c}(\omega)= & \frac{A_{2}(\omega) B_{1}(\omega)+A_{1}(\omega) B_{2}(\omega)}{2 A_{1}(\omega) A_{2}(\omega)}\left(X_{1}(\omega)+j X_{2}(\omega)\right) \\
& +\frac{A_{2}(\omega) B_{1}(\omega)-A_{1}(\omega) B_{2}(\omega)}{2 A_{1}(\omega) A_{2}(\omega)}\left(X_{1}(\omega)-j X_{2}(\omega)\right) \\
= & H_{\mathrm{cm}}(\omega) X_{c}(\omega)+H_{\mathrm{df}}(\omega) X_{c}^{*}(-\omega) .
\end{aligned}
$$

Equation (4) shows that the input complex signal $x_{c}(t)$ is processed in a parallel fashion by $h_{\mathrm{cm}}(t)$ and $h_{\mathrm{df}}(t)$ [Fig. 3(a)] [17] ${ }^{2}$. The common component of $H_{1}(\omega)$ and $H_{2}(\omega)$ forms $H_{\mathrm{cm}}(\omega)$ which gives the desired (direct) output $H_{\mathrm{cm}}(\omega) \cdot X_{c}(\omega)$. However, if $H_{1}(\omega)$ and $H_{2}(\omega)$ are not identical, i.e., gain $(\delta G(\omega))$ and phase mismatch $(\delta P(\omega))$ exist

\footnotetext{
${ }^{2}$ Originally, a similar model was proposed to describe a mismatched complex filter in [18]-[20].
}

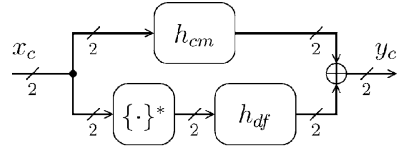

(a)

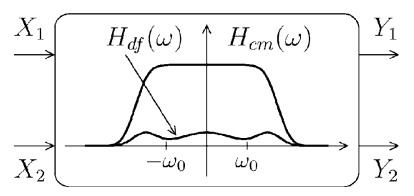

(b)

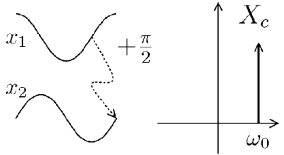

(c)

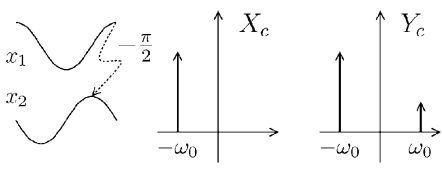

(d)

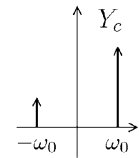

(a) Time-domain and (b) frequency-domain model of an imperfect two-path LPF. Imperfect filtering of a complex (c) positive-frequency and (d) negative-frequency input tone.

between them, then a nonzero $H_{\mathrm{df}}(\omega)$ contributes to a leakage (undesired or difference) output component $H_{\mathrm{df}}(\omega) \cdot X_{c}^{*}(-\omega)$.

For example, if a complex positive-frequency tone at $\omega_{0}$ undergoes an imperfect two-path filtering operation, then the complex output will contain, besides the desired component at $\omega_{0}$, a leakage component at $-\omega_{0}$ [Fig. 3(c)]. Similarly, a complex input tone at $-\omega_{0}$ will leak into $\omega_{0}$ [Fig. 3(d)]. Note that this distortion occurs independently from the leakage caused by the mixers/LO and the ADCs. In practical situation, all imperfections add.

\section{B. Butterworth Example}

As an illustrative example, a seventh-order Butterworth transfer function [21] with $8.8-\mathrm{MHz}$ bandwidth was chosen. Its magnitude response, phase response and group delay are shown in Fig. 4. The dots on the magnitude-response curve indicate the passband frequency components. This transfer function was implemented by a pair of active $G_{m}-C$ filters using cascade-of-poles topology [22]. The complex zero-IF filter was considered a two-input two-output linear system, and was modeled at circuit-element level (i.e., $G_{m}-s$ and $C-s$ ) using Simulink and Matlab. In an actual IC implementation, the values of circuit elements will show a discrepancy from their nominal values due to process variations, temperature changes and aging [22]. These fluctuations will alter the transfer function of the I path compared to the transfer function of the Q path, and cause I/Q imbalance, as described by (4) in mathematical terms.

To simulate this effect all circuit elements (i.e., $G_{m}-s$ and $C-s$ ) of the zero-IF two-path filter were perturbed by a normally-distributed mismatch of $1 \%$ variance; all sources of mismatches were assumed to be uncorrelated. Although this uniform mismatch is simplistic compared to a fabricated-IC scenario, it provides a first-order approximation and a good insight into the two-path filter's behavior. A mismatch of $\sigma=1 \%$ might be excessive, since an assumption of $3 \sigma=1 \%$ is more appropriate, but it would increase about $4 \times$ the simulation time. In conclusion, the relative IMR values claimed in this paper have more significance than the absolute IMR values.

Note that the simulations were performed using a black-box approach. In this method, a perfect quadrature complex signal, i.e., $x_{c}(t)=A \cos \left(\omega_{0} t\right)+j A \sin \left(\omega_{0} t\right)$, was applied to the input of the filter. The spectrum of the resulting complex output 

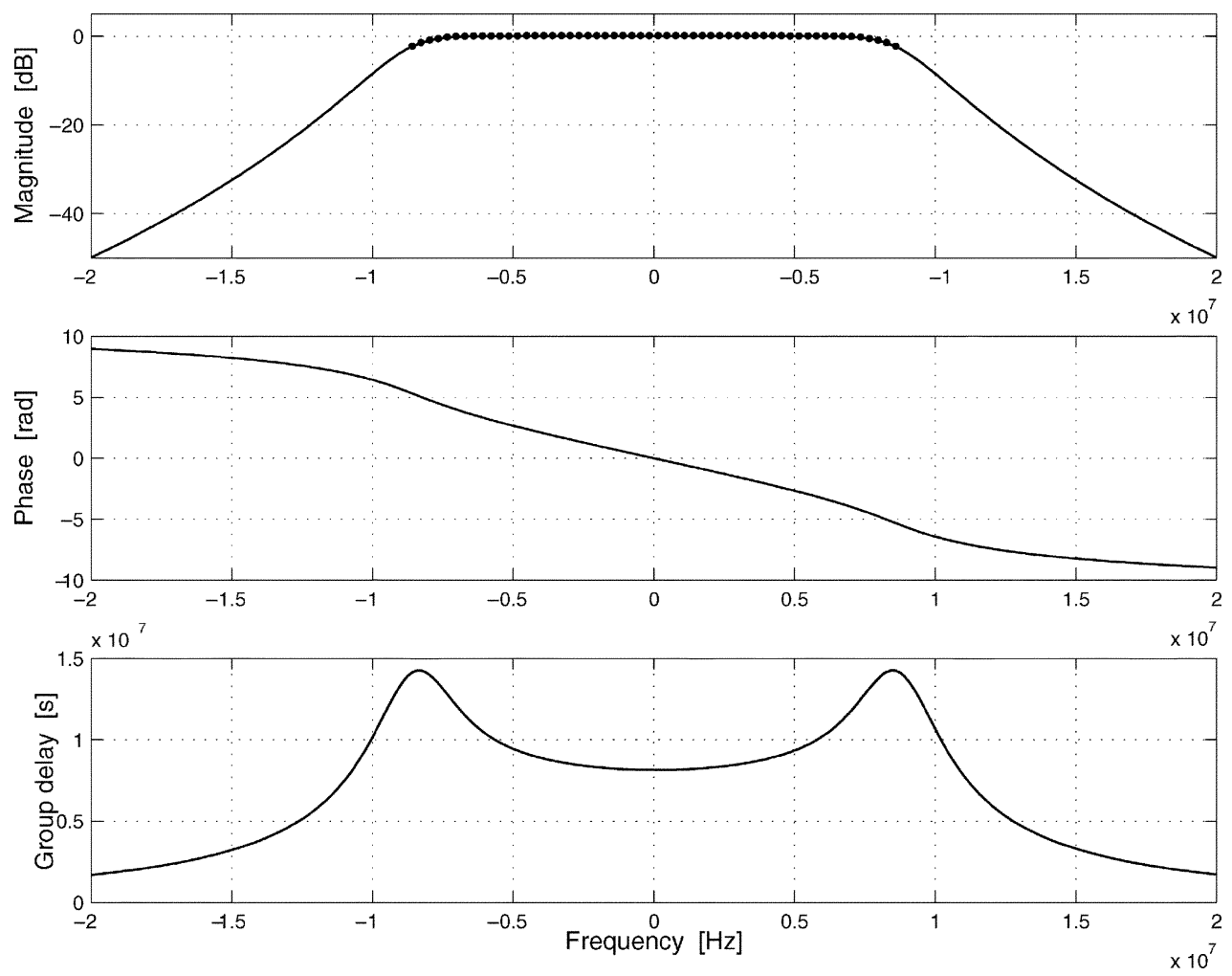

Fig. 4. Seventh-order 8.8-MHz Butterworth filter.

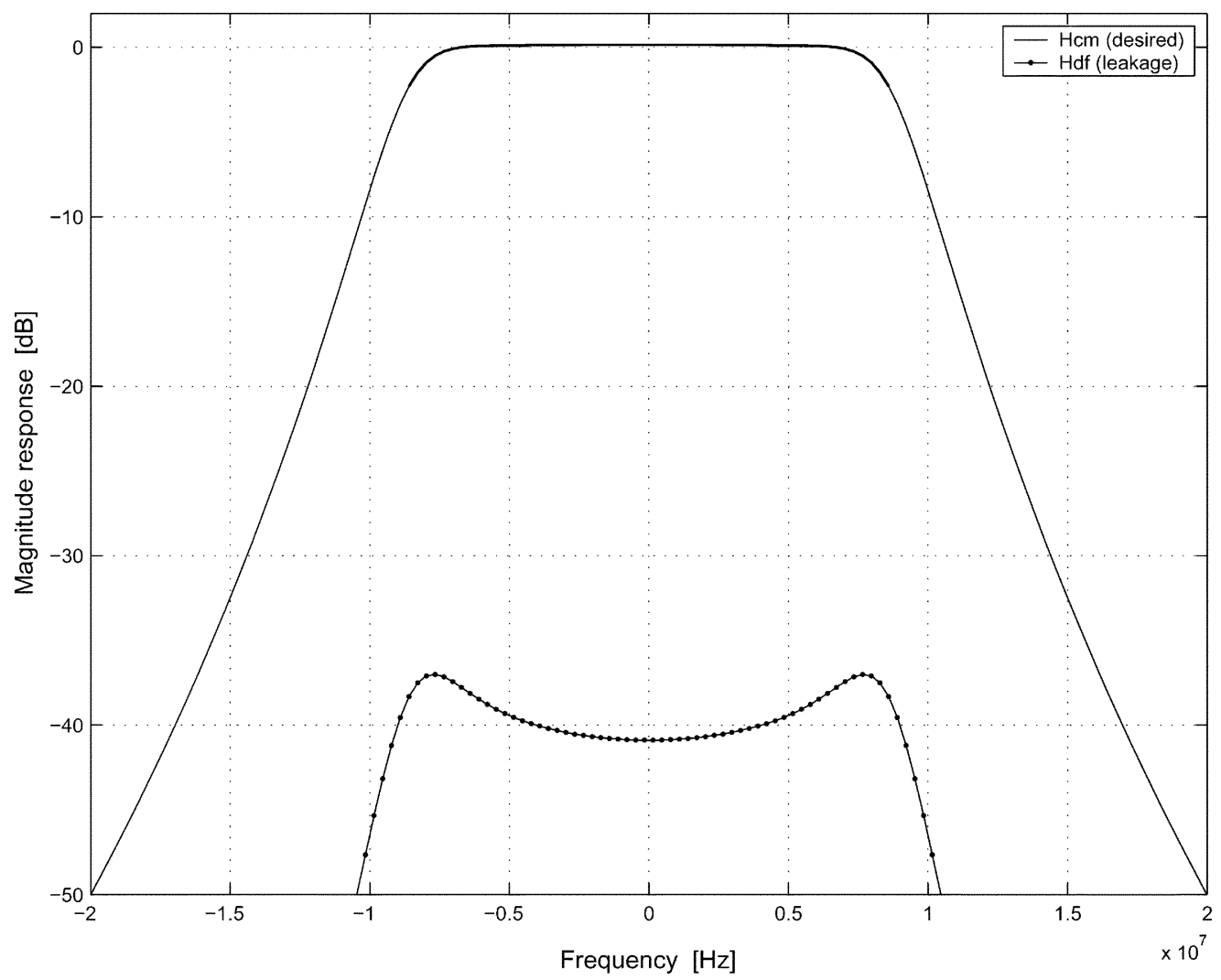

Fig. 5. Desired and leakage responses.

$y_{c}(t)$ was measured at $\omega_{0}$ and $-\omega_{0}$, providing the values for $H_{\mathrm{cm}}\left(\omega_{0}\right)$ and $H_{\mathrm{df}}\left(-\omega_{0}\right)$, respectively (Fig. 3). The experiment was performed for the range of frequencies of interest, i.e., -20 to $20 \mathrm{MHz}$.
Since the image rejection ratio

$$
\operatorname{IMR}(\omega)=20 \log _{10}\left|\frac{H_{\mathrm{cm}}(\omega)}{H_{\mathrm{df}}(\omega)}\right| \quad \mathrm{dB}
$$




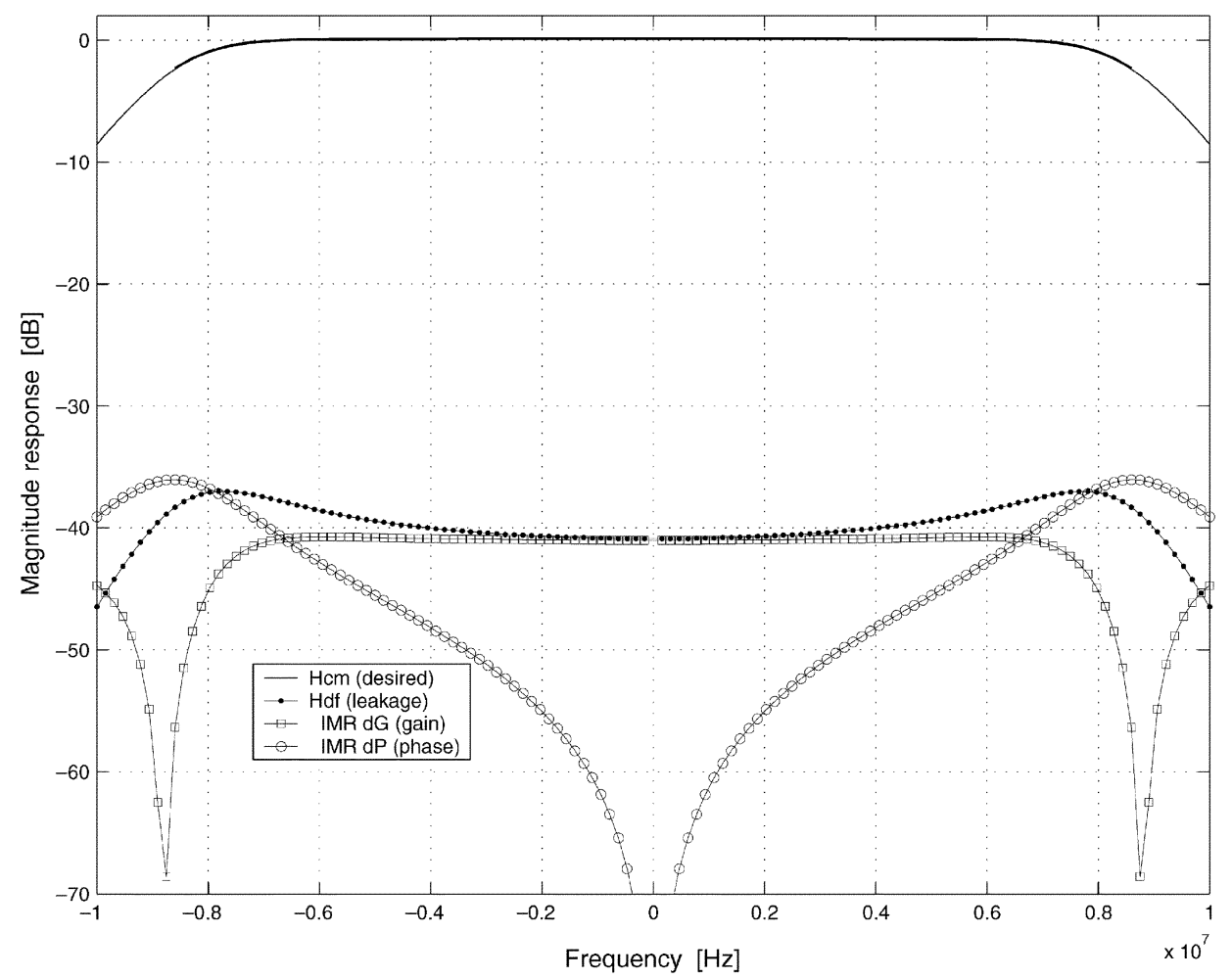

Fig. 6. I/Q leakage contributors: gain and phase mismatch.

is a function of frequency, it is convenient to calculate for passband frequencies $(\omega \in \mathrm{BW})$ its rms average

$$
\mathrm{IMR}_{\mathrm{rms}}=10 \log _{10} \int_{\omega \in \mathrm{BW}} \frac{1}{\mathrm{BW}} \cdot\left|\frac{H_{\mathrm{cm}}(\omega)}{H_{\mathrm{df}}(\omega)}\right|^{2} d \omega \quad \mathrm{dB}
$$

and its minimum

$$
\mathrm{IMR}_{\min }=\min \{\operatorname{IMR}(\omega)\}_{\omega \in \mathrm{BW}} \quad \mathrm{dB}
$$

values.

The resulting desired and undesired responses are illustrated on Fig. 5. It turns out that the shape of the leakage $\left|H_{\mathrm{df}}(\omega)\right|$ resembles the shape of the group delay (Fig. 4). This suggests that the phase error may be the dominant I/Q imbalance contributor.

\section{Gain and Phase Errors}

To investigate the "group-delay-like" leakage, the frequencydependent $\operatorname{IMR}(\omega)$ was decomposed ${ }^{3}$ into gain $(\delta G(\omega))$ and phase $(\delta P(\omega))$ errors (Fig. 6), since these errors are orthogonal $[5] ;\left.\mathrm{IMR}_{\delta G} \doteq \mathrm{IMR}\right|_{\delta P=0}$ and $\left.\mathrm{IMR}_{\delta P} \doteq \mathrm{IMR}\right|_{\delta G=0}$, where $\operatorname{IMR}(\delta G, \delta P)$ is given by (2).

Fig. 6 shows that both gain and phase errors contribute to the total $\operatorname{IMR}(\omega)$. Phase errors dominate the $\operatorname{IMR}(\omega)$ near the edge of the pass band, while gain errors are approximately flat and they are the dominant $\operatorname{IMR}(\omega)$ contributor around dc, i.e., at low frequencies. (Note that the total $\operatorname{IMR}(\omega)$ itself is not shown on Fig. 6 for simplicity; it can be calculated from (5).) These are just partial results. Do these observations reveal a deterministic trend?

\footnotetext{
${ }^{3} \mathrm{~A}$ discussion of zero-IF versus low-IF filters can be found in Appendix II.
}

\section{Statistical Analysis}

In order to draw general conclusions, the experiment was repeated for 2000 mismatch states (i.e., 2000 trials or 2000 realizations of the random mismatch process) and the results were processed statistically. First, the $\operatorname{IMR}(\omega)$ is investigated as a function of frequency. The $\operatorname{IMR}(\omega)$ curves resulted from the 2000 trials are shown in Fig. 7 on top of each other forming a gray "background." The $\operatorname{IMR}(\omega)$ curves were obtained using 112 complex passband test tones. Therefore, the $\operatorname{IMR}(\omega)$ curves can be "sliced" into 112 frequency bins; each of them contains 2000 statistical IMR values. The histogram of each frequency bin was calculated, thus the median $(50 \%)$, the $1 \sigma(65.87 \%)$ and $3 \sigma(99.74 \%)$ yield values were determined and plotted on Fig. 7. The distributions were not exactly Gaussian, so the "median" was considered a more accurate average than the "mean."

The statistical passband $\operatorname{IMR}(\omega)$ can also be decomposed into gain and phase contributors. The resulting median yield curves, as a function of frequency, are shown in Fig. 8. Again, phase errors dominate the $\operatorname{IMR}(\omega)$ near the edge of the pass band, while gain errors are approximately flat and they are the dominant $\operatorname{IMR}(\omega)$ contributor at low frequencies. Additionally, the phase error resembles a plain delay and the gain error is more-or-less constant! This suggests that a simple I/Q compensation scheme is feasible-described next.

\section{E. Delay Error}

Fig. 9 quantifies the validity of the delay approximation of the statistical phase error. Actually, both $\tau_{1} \cdot \omega$ and $\tau_{2} \cdot \omega$ approximate $\delta P(\omega)$ reasonably well. $\tau_{1}$ was obtained by estimating the delay between the two filters at $f_{p}=8 \mathrm{MHz}$, so

$$
\tau_{1}=\frac{\hat{\delta P}\left(f_{p}\right)}{2 \pi f_{p}}=\frac{\hat{\delta P}\left(\omega_{p}\right)}{\omega_{p}} \quad \mathrm{dgr} .
$$




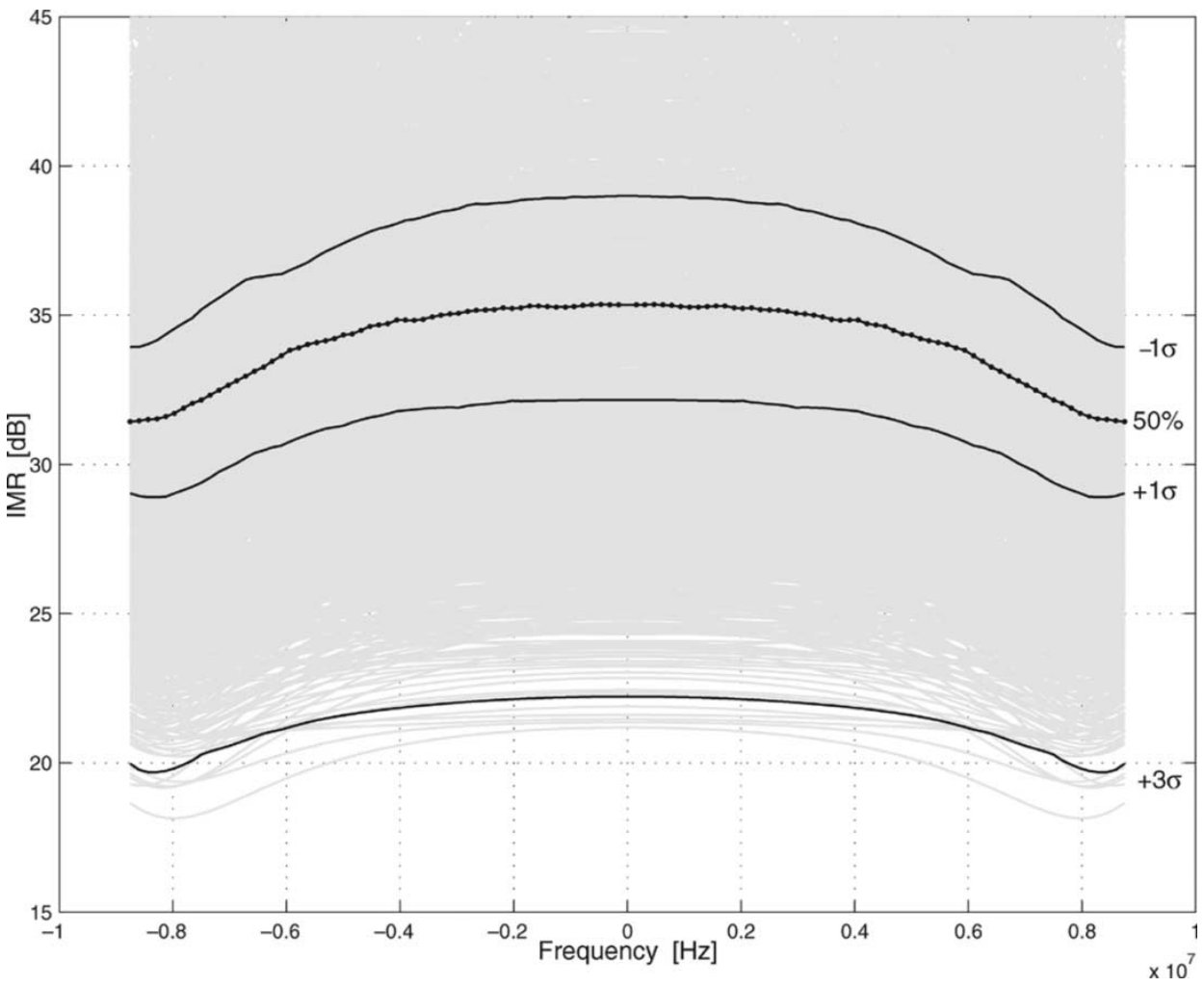

Fig. 7. Statistical response of the total I/Q leakage.

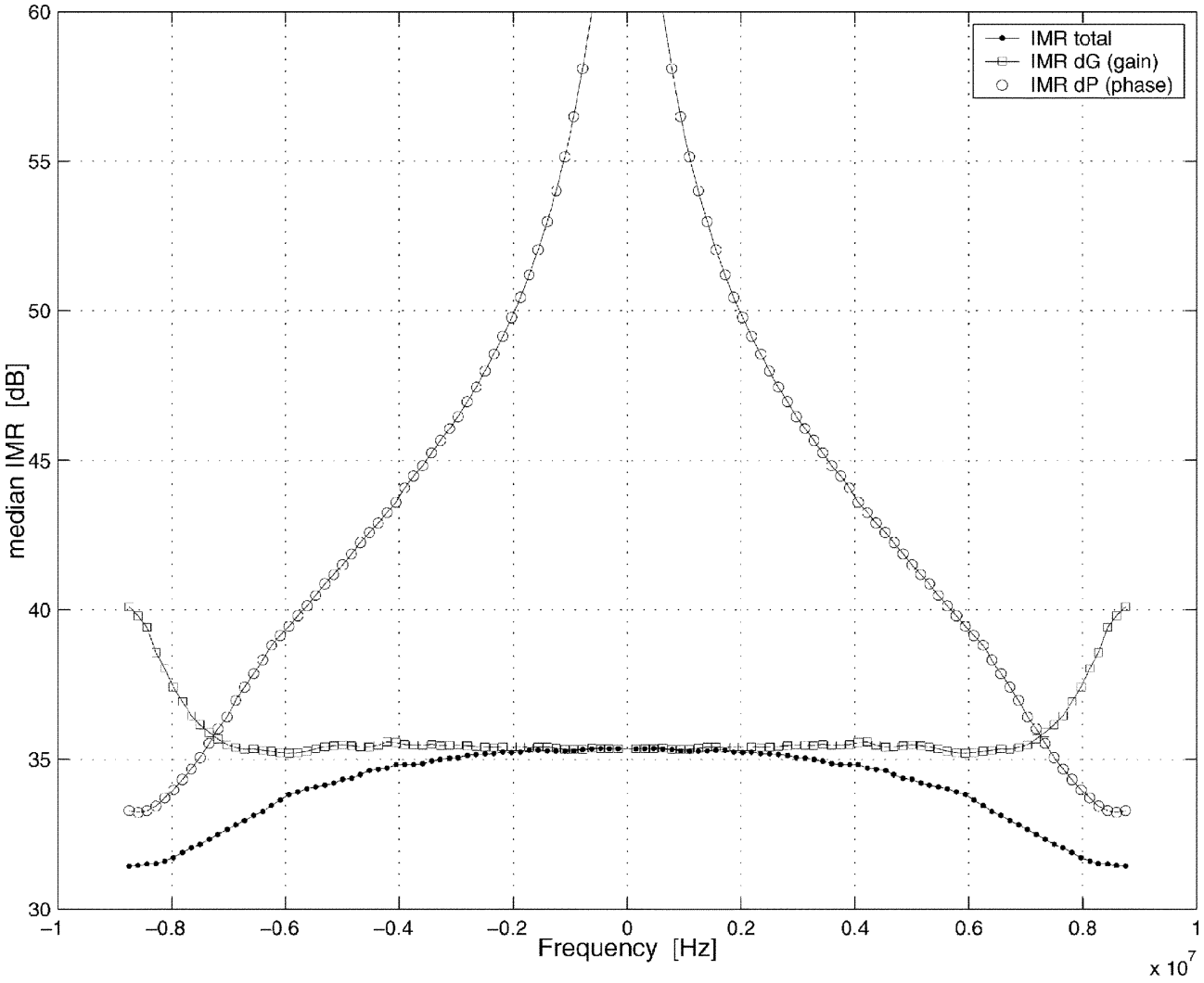

Fig. 8. Statistical response of $\mathrm{I} / \mathrm{Q}$ leakage contributed by gain and phase mismatch.

Since the delay estimate is a first-order linear approximation of a frequency-dependent phase error, an optimal line can be found by minimizing the rms value of the estimation error, i.e., $\epsilon(\omega)=\delta P(\omega)-\delta \hat{P}(\omega)$, by choosing various values for $f_{p}$.
It turns out that this occurs at $f_{p} \cong 7 \mathrm{MHz}$ for the assumed filter (Fig. 10). Therefore, for the best rms $\epsilon(\omega), f_{p} \cong 7 \mathrm{MHz}$ should be used. However, this estimation gives large errors near the passband edge (Fig. 9), where the phase error is dominant 

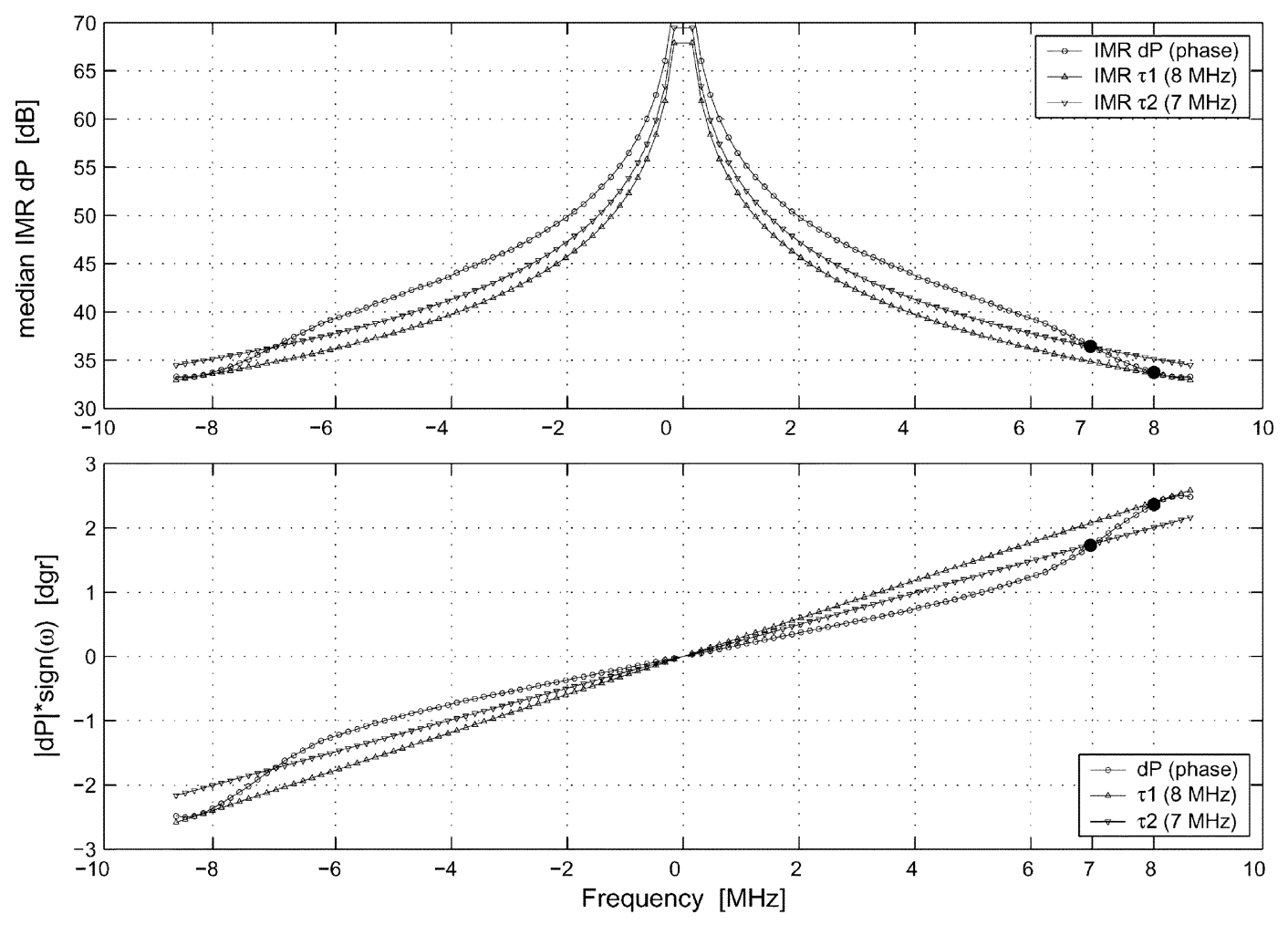

Fig. 9. Delay approximation of phase imbalance.

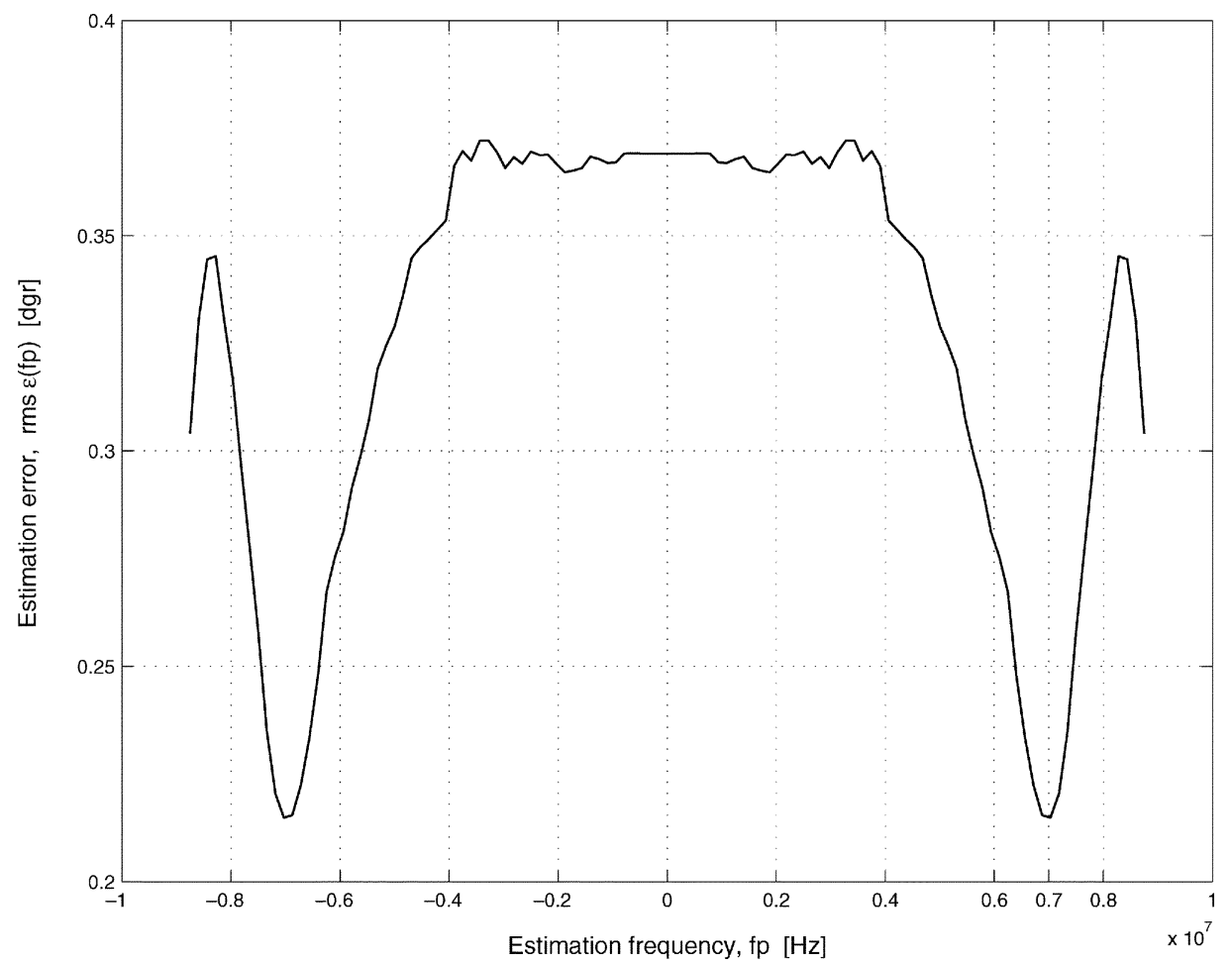

Fig. 10. Estimation error of the linear approximation of phase imbalance.

(Fig. 8). On the other hand, when the delay is estimated at $f_{p}=$ $8 \mathrm{MHz}$, then, the $\epsilon(\omega)$ will improve near the passband edge, but the rms average of $\epsilon(\omega)$ will suffer. This tradeoff between good passband edge $\epsilon(\omega)$ and low rms $\epsilon(\omega)$ will drive the choice of $f_{p}$ depending on the application.

\section{I/Q MisMATCH ESTIMATION AND CORRECTION}

Based on the previously described I/Q mismatch analysis, a delay-based I/Q compensation scheme is proposed in Fig. 11. In the one-step estimation procedure a two-tone test signal is 

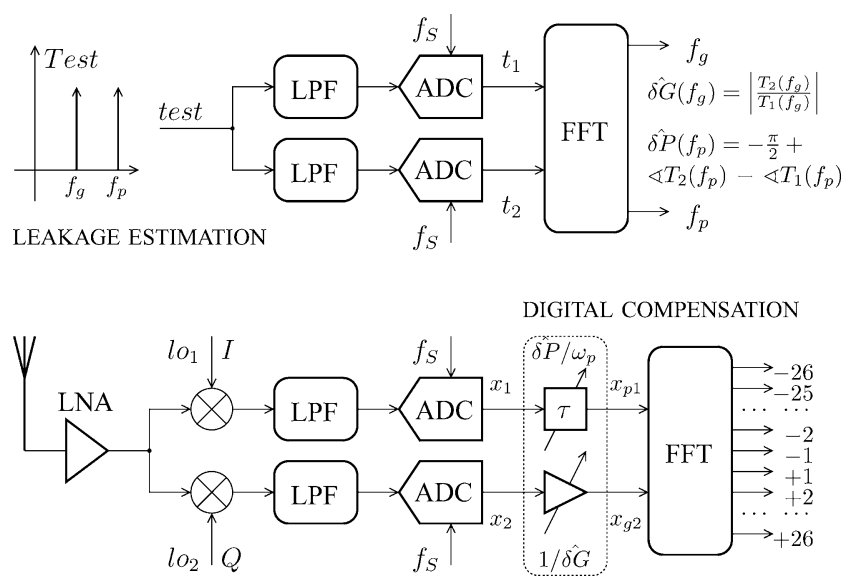

Fig. 11. Proposed I/Q estimation (top) and compensation (bottom) scheme.

injected to the input of both LPFs. Next, the estimated gain error $\hat{\delta} G$ and phase error $\delta \hat{P}$ are determined from the digital outputs $t_{1}$ and $t_{2}$ of the ADCs, measured at $f_{g}$ and $f_{p}$, respectively

$$
\left\{\begin{array}{l}
\delta \hat{G}\left(f_{g}\right)=\left|\frac{T_{2}\left(f_{g}\right)}{T_{1}\left(f_{g}\right)}\right| \\
\delta \hat{P}\left(f_{p}\right)=\varangle T_{2}\left(f_{p}\right)-\varangle T_{1}\left(f_{p}\right)-\frac{\pi}{2} .
\end{array}\right.
$$

The two frequencies of $f_{g}$ and $f_{p}$ should be positioned at the middle and at the edge of the pass band of the filter, respectively. For example, choosing $f_{g}=5 \mathrm{MHz}$ and $f_{p}=7 \mathrm{MHz}$ is a possible scenario-as explained earlier.

Since both $\delta \hat{G}$ and $\delta \hat{P}$ are determined by comparing the relative difference between $t_{1}$ and $t_{2}$ (Fig. 11), the amplitude and frequency accuracy of the injected two-tone test signal are not critical. Note that the test signal is a real one-wire, baseband signal, so there is not a need to generate precise quadrature $\mathrm{I} / \mathrm{Q}$ calibration tones. For a transceiver, in general, the TX DACs could generate calibration signals for the RX filters, and the RX ADCs could be used to calibrate the TX filters-without relying on the I/Q matching of the calibrating hardware. Finally, the frequency-independent I/Q imbalance of the high-frequency front-end (i.e., LO and mixers) is not addressed by the proposed method; it should be separately corrected by using, e.g., [5], [8], [9], [11], [13].

The proposed digital compensation, shown on the bottom of Fig. 11, contains a tunable delay and a tunable gain in the I and $\mathrm{Q}$ paths, respectively. Since the compensation is done in the time domain (i.e., before the FFT), frequency-offset errors between the TX and RX LO, allowed in 802.11a [4], do not affect the correction.

The tunable delay can be well approximated by a first-order interpolation filter [Fig. 12(a)] since the delay mismatch is expected to be small compared to the sampling period $T_{S}=1 / f_{S}$. The structure of the delay filter is derived in Appendix III, and the resulting conceptual digital correction circuitry is shown in Fig. 12(b). It uses two multipliers with tunable coefficients and two adders. Simulations presented in this paper use a floatingpoint arithmetic to implement the mismatch estimation and digital correction; fixed-point simulations of the proposed method will be provided soon.

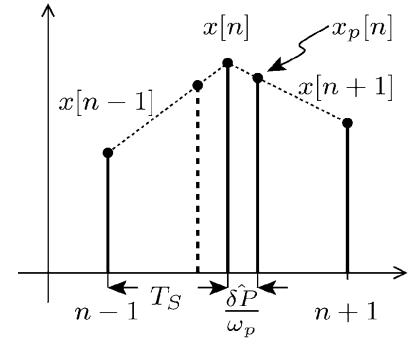

(a)

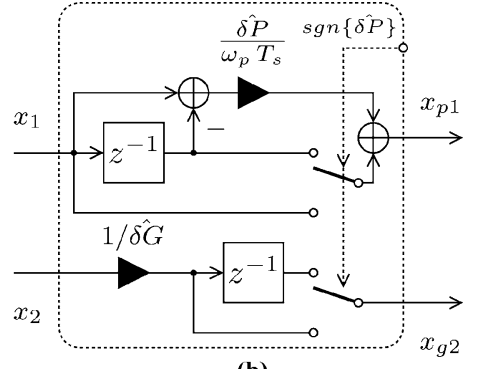

(b)
Fig. 12. Implementation. (a) Variable delay (first-order interpolation). (b) Digital correction circuitry.

\section{A. Butterworth Example}

Simulation results are presented next. First, the proposed delay-based I/Q correction is applied to one scenario of mismatched pair of Butterworth filters [21], shown in Fig. 6. When the gain is estimated at $f_{g}=5 \mathrm{MHz}$ and the delay is estimated at $f_{p}=8 \mathrm{MHz}$, the correction improves the $\operatorname{IMR}(\omega)$ significantly (Fig. 13). Since the gain error is not flat near the passband edge, the gain correction introduces errors. Therefore, the corrected $\operatorname{IMR}(\omega)$ degrades for these frequencies. Similarly, the delay approximation is not accurate for low frequencies, so the corrected $\operatorname{IMR}(\omega)$ will suffer from these residual errors.

Another possibility is to determine the delay at the optimal frequency of $f_{p}=7 \mathrm{MHz}$ (Fig. 10) and, again, estimate the gain at $f_{g}=5 \mathrm{MHz}$ (Fig. 14). In this case, the rms average of the residual phase error will be smaller. Therefore, the corrected $\operatorname{IMR}(\omega)$ improves for low frequencies (Fig. 14) compared to the previous scenario (Fig. 13). However, it will be worse near the passband edge.

The proposed delay-based correction was applied for all 2000 trials analyzed earlier (Fig. 7). The median $\operatorname{IMR}(\omega)$ of these trials is shown in Fig. 15 for uncorrected and corrected filters. Two frequencies were used for delay estimation: $f_{p}=8 \mathrm{MHz}$ and $f_{p}=7 \mathrm{MHz}$. After correction, once again, the 8-MHz estimation leads to better passband minimum IMR (7), while $7 \mathrm{MHz}$ gives better passband rms IMR (6). The gain was estimated at $f_{g}=5 \mathrm{MHz}$.

The extensive statistical analysis presented earlier allows determining the yield of such uncompensated/compensated filters. The yield for passband rms IMR and passband minimum IMR are shown in Fig. 16 for the uncompensated and two compensated scenarios. When $f_{p}=8 \mathrm{MHz}$ is used, the rms IMR is improved by about $9 \mathrm{~dB}$ for both median and $3 \sigma$ yields. For $f_{p}=7 \mathrm{MHz}$ the rms IMR improvement is even better (i.e., about $10 \mathrm{~dB}$ ), but, as expected, the min IMR gets worse by about $1 \mathrm{~dB}$ compared to $f_{p}=8-\mathrm{MHz}$ case.

\section{B. Various Filters. Discussion}

The proposed method, a priori designing the correction, identifies the expected gain and phase imbalance based on an extensive statistical analysis. As shown earlier, these results may confirm a frequency-independent passband gain error and a linear passband phase error (i.e., a delay) for the pair of filters. In other words

$$
\begin{gathered}
\left.\delta G(\omega)\right|_{\omega \in \mathrm{BW}}=\delta G=\text { constant } \\
\left.\frac{\partial \delta P(\omega)}{\partial \omega}\right|_{\omega \in \mathrm{BW}}=\tau=\mathrm{constant} .
\end{gathered}
$$




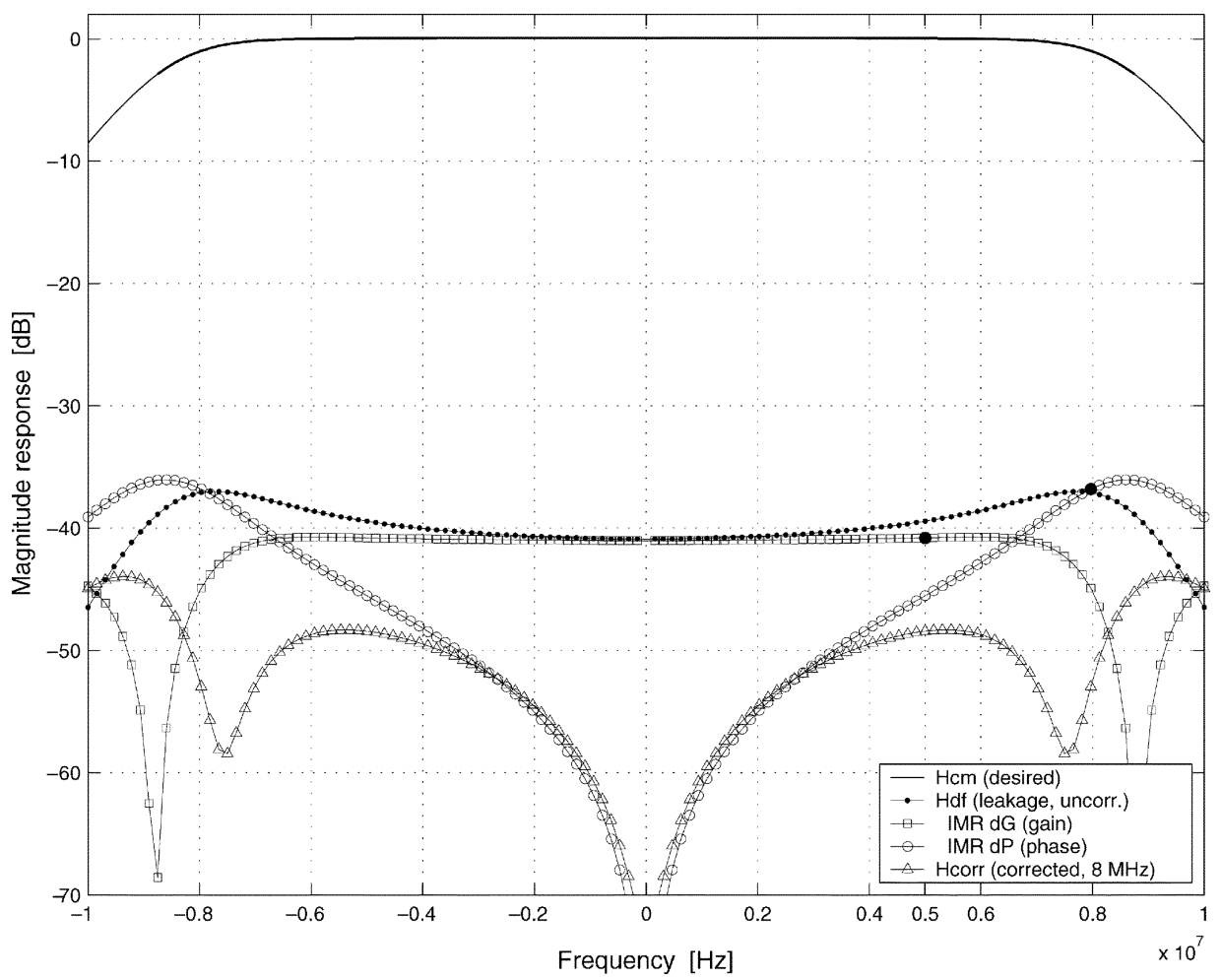

Fig. 13. One scenario of delay-based I/Q compensation with $f_{p}=8 \mathrm{MHz}$.

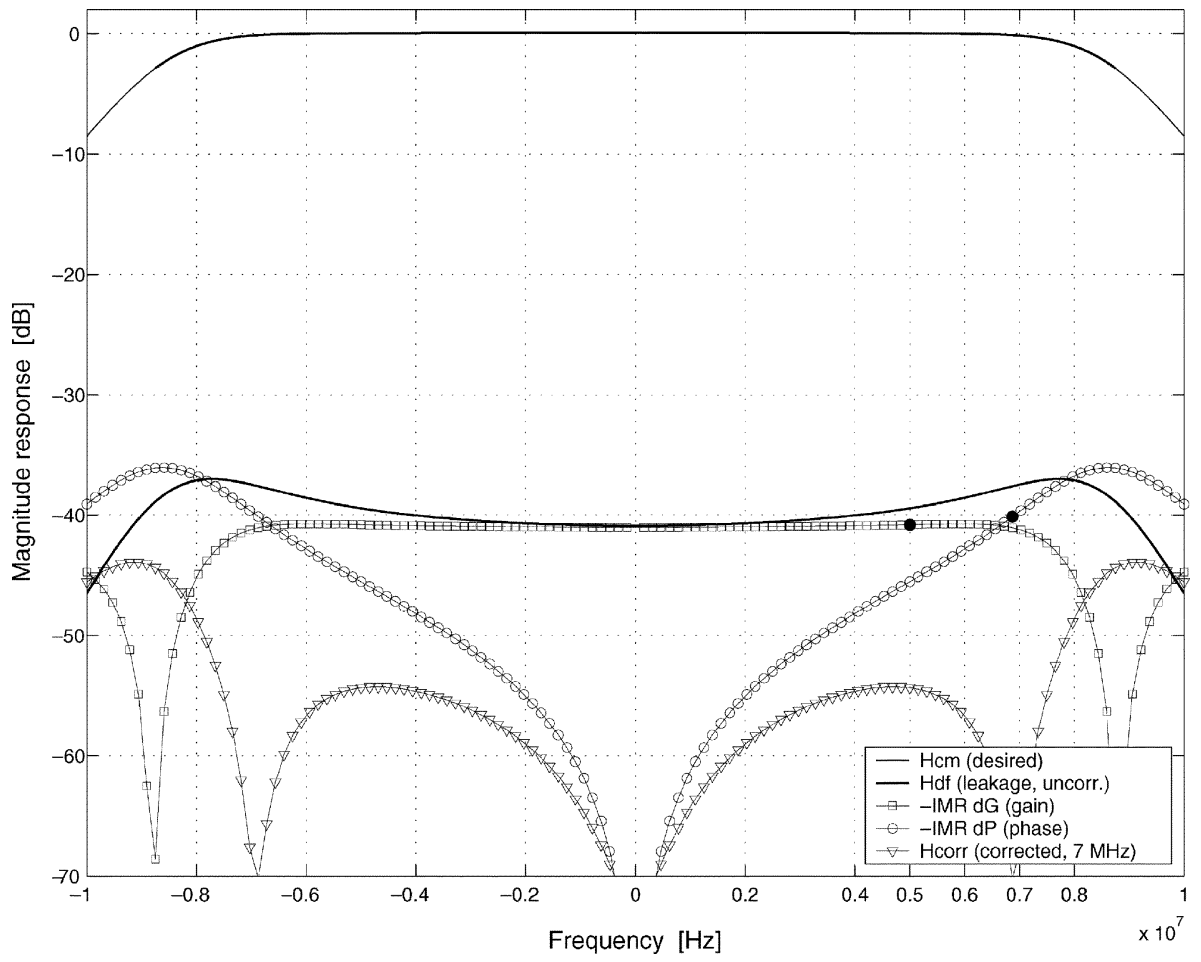

Fig. 14. One scenario of delay-based I/Q compensation with $f_{p}=7 \mathrm{MHz}$.

For filters where these strict assumptions hold, the proposed I/Q compensation has the advantage that it provides a fast-estimation (noniterative) and hardware-efficient correction method. However, the proposed method relies on these assumptions, so it is not effective for filters with different behavior.
The cascade-of-pole Butterworth filter, described in previous sections, significantly benefitted from the delay-based correction. In order to check the validity of the proposed method for other filters, first, the same Butterworth transfer function was implemented by a ladder topology [21], [22]. In addition, a 


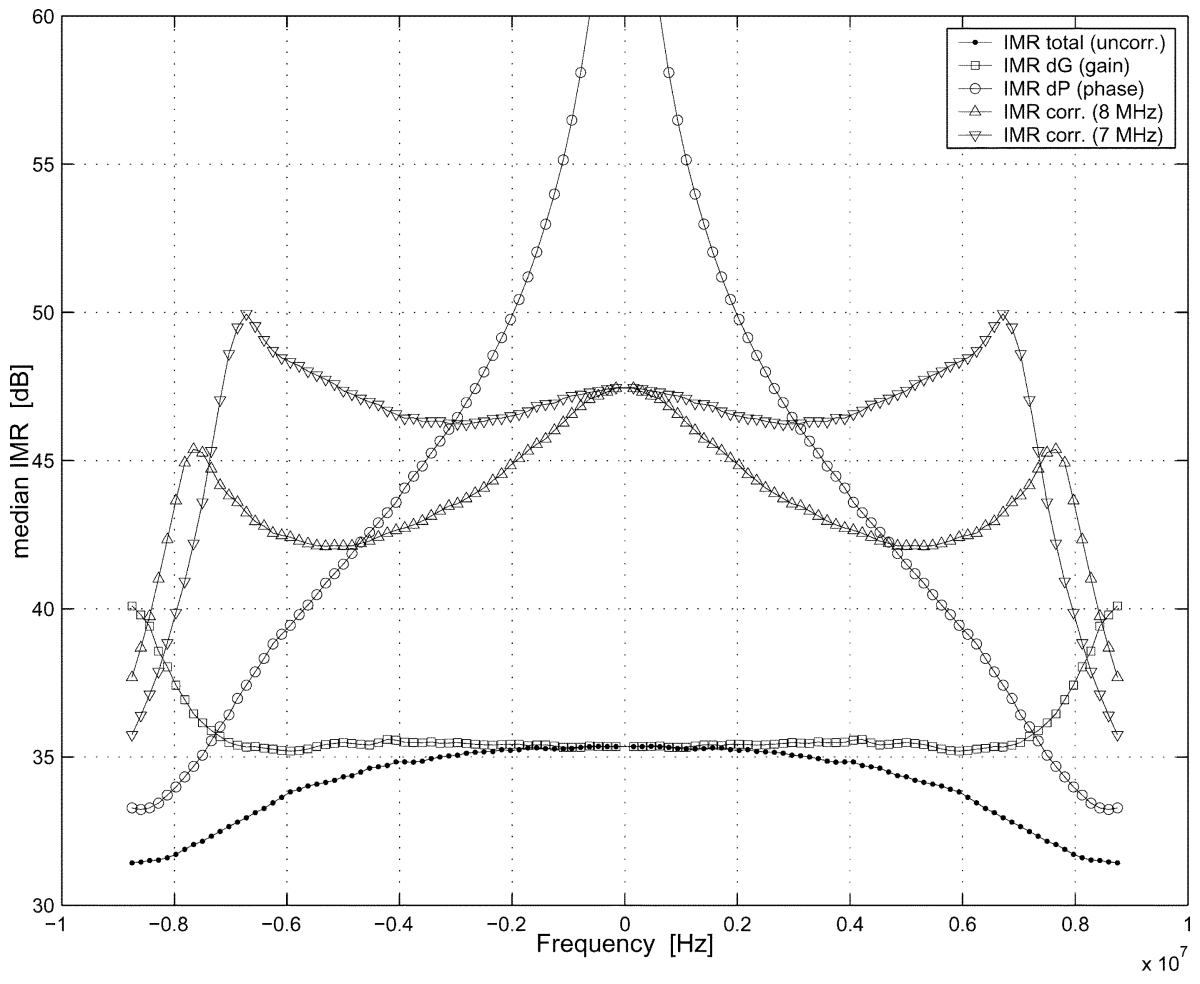

Fig. 15. Statistical results of delay-based I/Q compensation.

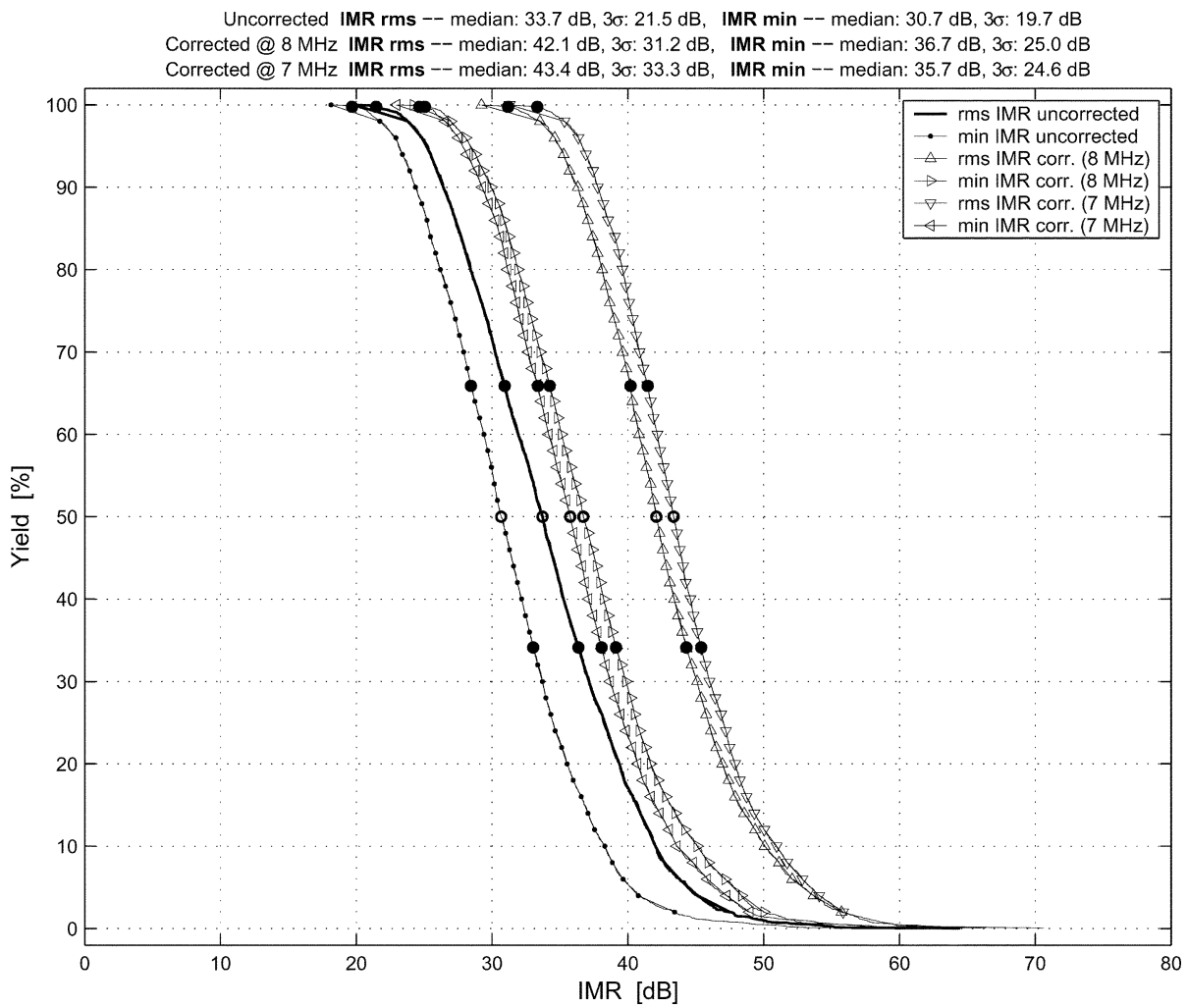

Fig. 16. Yield curves for delay-based I/Q compensation.

seventh-order 0.5-dB ripple 8.8-MHz wide Chebyshev transfer function [21] was investigated for both cascade-of-poles and ladder topologies.
Fig. 17 shows the frequency dependence of the uncompensated/compensated median $\operatorname{IMR}(\omega)$ and gain/phase errors based on a 2000-trial statistical analysis for these four filter 

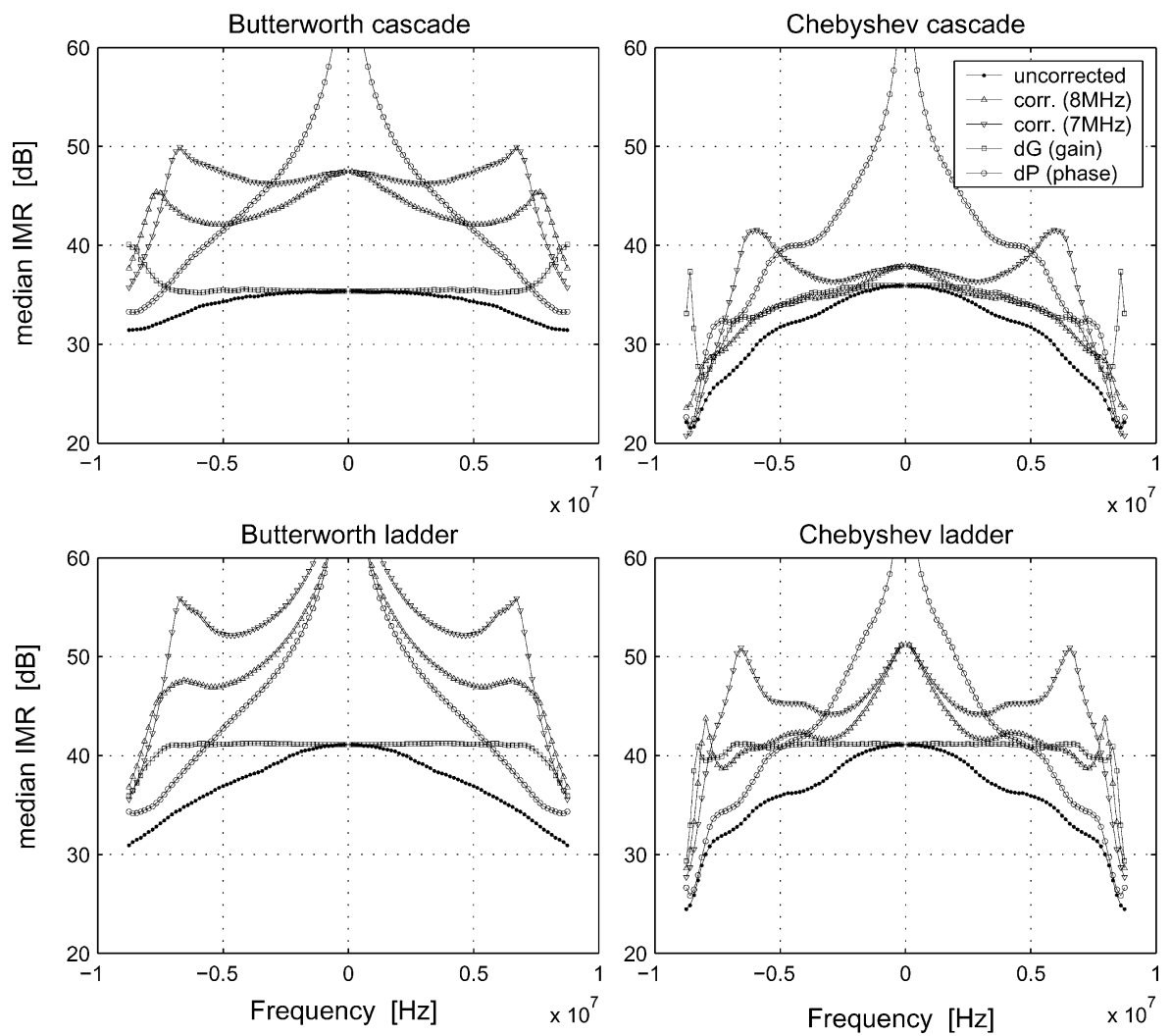

Fig. 17. Statistical results for various filters.
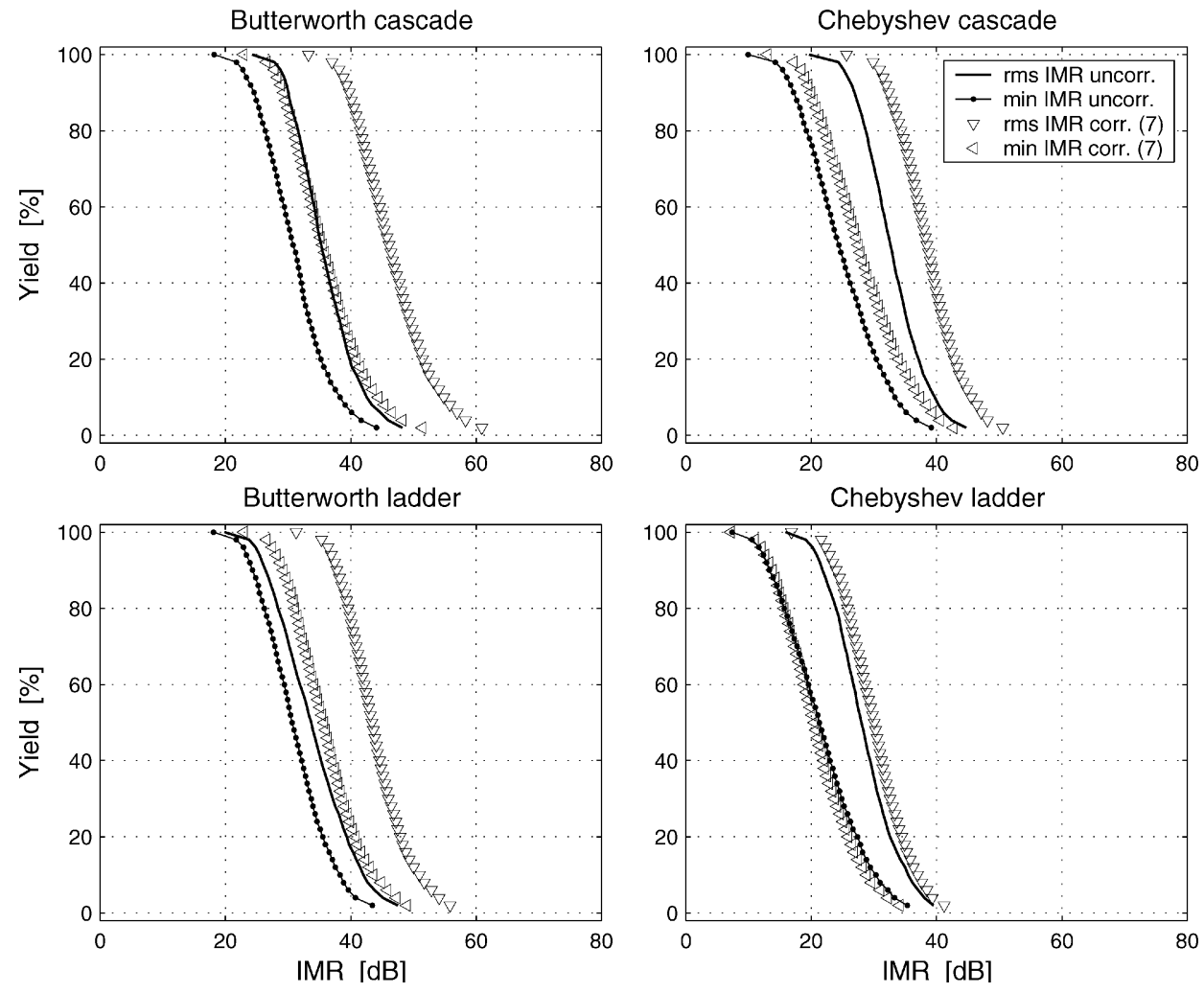

Fig. 18. Yield curves for various filters.

types. The corresponding yield curves are shown in Fig. 18. In addition, the optimal $f_{p}$ frequency can be determined based on Fig. 19. Finally, Fig. 20 summarizes the results.
The uncompensated $\operatorname{IMR}(\omega)$ results will be analyzed first. Using Butterworth over Chebyshev transfer functions improves the amount of the rms IMR by about 3-5 dBs (Fig. 20). Also, 

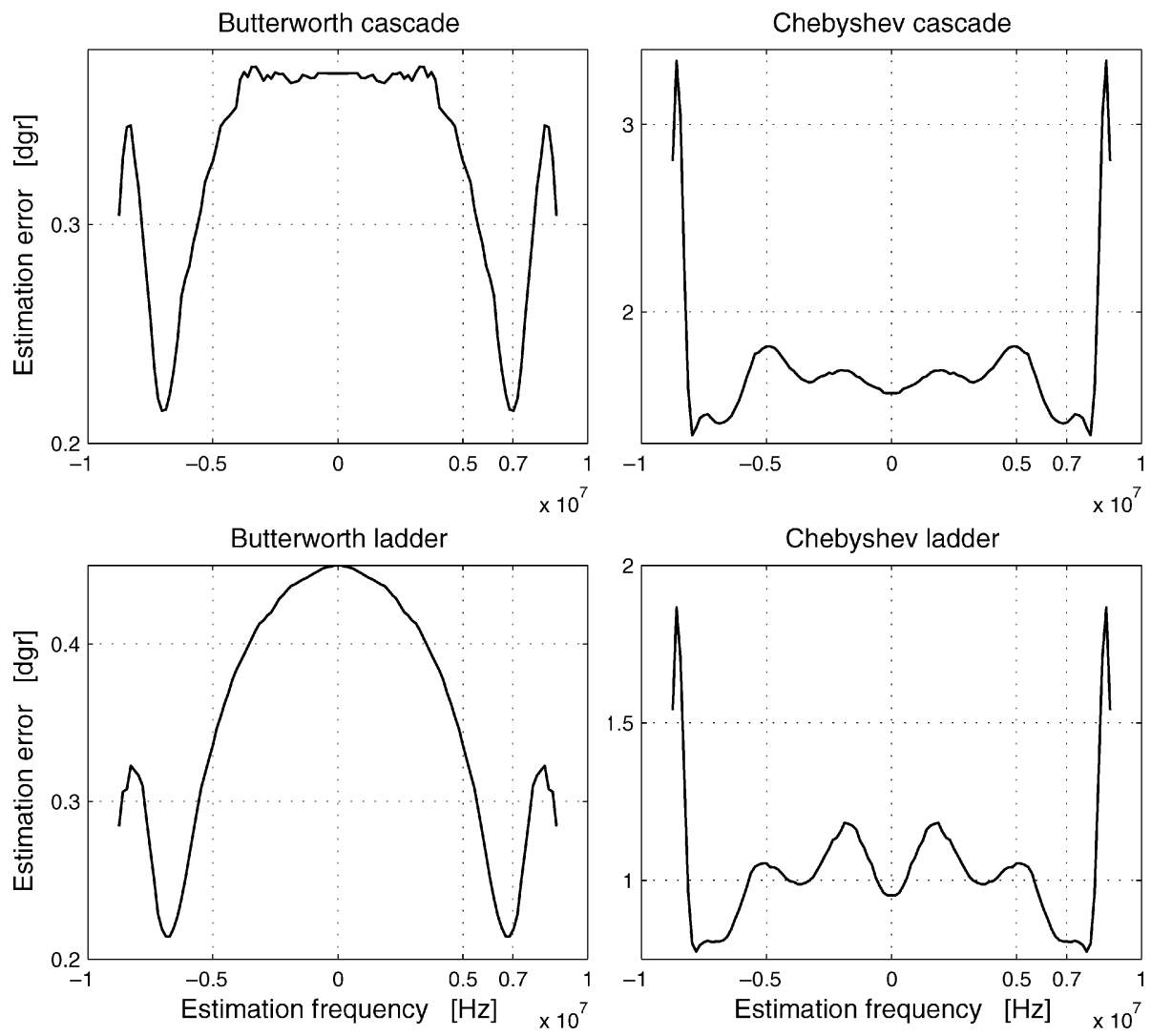

Fig. 19. Estimation error for various filters.

\begin{tabular}{|c|c|c|c|c|c|c|}
\hline \multirow{3}{*}{ T } & \multirow{3}{*}{\multicolumn{2}{|c|}{$\begin{array}{c}\text { rms IMR [dB] } \\
\mathrm{fp}=7, \mathrm{fg}=5[\mathrm{MHz}]\end{array}$}} & \multicolumn{4}{|c|}{ TRANSFER FUNCTION } \\
\hline & & & \multicolumn{2}{|c|}{ Butterworth } & \multicolumn{2}{|c|}{ Chebyshev } \\
\hline & & & median & $3 \sigma$ & median & $3 \sigma$ \\
\hline 0 & \multirow{3}{*}{ Cascade } & leakage & 33.7 & 21.5 & 28.0 & 17.5 \\
\hline o & & corrected & 43.4 & 33.3 & 30.1 & 19.6 \\
\hline $\mathrm{L}$ & & $\Delta$ & 9.7 & 11.8 & 2.1 & 2.1 \\
\hline $\mathrm{O}$ & \multirow{3}{*}{ Ladder } & leakage & 35.2 & 25.7 & 32.7 & 21.8 \\
\hline G & & corrected & 46.1 & 35.0 & 38.5 & 26.9 \\
\hline$Y$ & & $\Delta$ & 10.9 & 9.3 & 5.8 & 5.1 \\
\hline
\end{tabular}

Fig. 20. Summary of results.

the use of a ladder topology yields to about 2-4-dB better rms IMR than cascade-of-poles topology; this, indeed, confirms the reduced sensitivity to circuit-element mismatch of ladder filters [22]. Interestingly, the gain error of the cascade-of-poles Chebyshev gradually bends over frequency (Fig. 17); so, the constant gain-error assumption does not hold well. However, the gain error of a ladder Chebyshev flattens out except a sharp increase of about $10 \mathrm{~dB}$ close to the passband edge. Butterworth filters show an approximately flat gain-error response with a slight bend near the passband edge.

Fig. 19 shows that the best phase-error estimation occurs for $f_{p}$ of about $7 \mathrm{MHz}$ for all four filters. Although there is a global minima at about $8 \mathrm{MHz}$ for the Chebyshev transfer function, the local minima at about $7 \mathrm{MHz}$ is not significantly higher and, more importantly, is much flatter. Therefore, $f_{p}=7 \mathrm{MHz}$ will be used for all filters for "best" rms phase-error estimation. Note that Butterworth filters introduce roughly $5 \times$ less rms estimation error than Chebyshev filters for $f_{p}=7 \mathrm{MHz}$ (i.e., $\sim 0.2^{\circ}$ versus $\sim 1^{\circ}$, Fig. 19). Therefore, Butterworth filters are much better candidates for delay-based compensation due to their maximally-flat response than their ripply, but more selective, Chebyshev counterparts.

The effectiveness of the proposed compensation can be determined from Figs. 17, 18 and 20. Clearly, a pair of ladder Butterworth filters is the best choice. They are the least affected by circuit-element mismatch to start with (i.e., uncorrected median rms IMR $=35.2 \mathrm{~dB}$, Fig. 20), and they can be the most effectively corrected by the proposed delay-based correction (i.e., corrected median rms IMR $=46.1 \mathrm{~dB}$ ). Both the cascade-of-poles Butterworth and ladder Chebyshev filters significantly benefit from the correction, which improves their performance by about $6-10 \mathrm{~dB}$ (Fig. 20). However, the approximately 2-dB improvement for a cascade-of-poles Chebyshev filter may be inadequate to warrant their compensation.

To ease the analog filtering requirements, oversampling may be used in the TX and/or RX chain at the expense of using faster data converters. In that case, the filter's bandwidth may be slightly increased by, e.g., $10 \%$ or $20 \%$, on the expense of some selectivity loss. From Fig. 17, results that opening up the filter has the advantage that it reduces the uncompensated I/Q imbalance affecting the actual signal. In addition, the compensated IMR improves even more. For example, the compensated median rms IMR of ladder Butterworth filters increases by 4.9 and $8.2 \mathrm{~dB}$ for $10 \%$ and $20 \%$ bandwidth increase, respectively, while the uncorrected median rms IMR gets improved by only $1.7 \mathrm{~dB}$ for the 20\% bandwidth stretching (Fig. 17). Both Chebyshev 


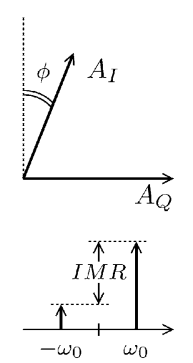

(a)
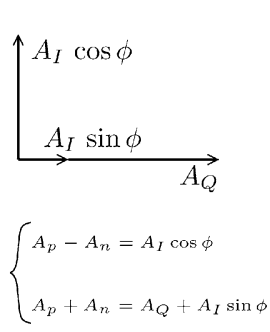

(b)

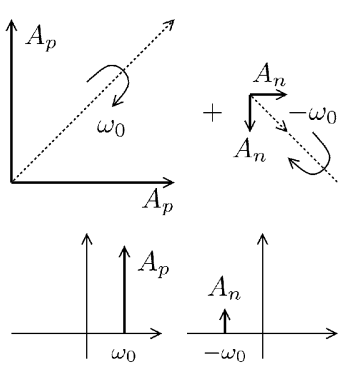

(c)
Fig. 21. Gain and phase mismatch in quadrature signals.

filters show about 6-dB compensated and 3-dB uncompensated IMR improvement for $20 \%$ bandwidth increase. These values are 3-dB and $0.7 \mathrm{~dB}$ for the cascade-of-pole Butterworth case.

Based on the analysis presented in this paper, a "backward thinking" filter-design methodology may emerge. A systemlevel designer should consider the I/Q imbalance as a constraint (like the stop-band attenuation, bandwidth or group delay) in choosing the transfer function and the topology. By making a choice which is a priori favorable for the proposed delay-based compensation (e.g., ladder Butterworth) a simple and effective $\mathrm{I} / \mathrm{Q}$ compensation is possible. When the I/Q-imbalance constraint is ignored, laborious and costly frequency-dependent I/Q correction schemes [10], [12], [14], [15] are inevitable.

\section{CONCLUSION}

In this paper, a delay-based I/Q compensation was proposed based upon an extensive statistical I/Q-mismatch analysis. The frequency-dependent I/Q imbalance was decomposed into gain-error and phase-error contributors. It turns out that, usually, the statistical gain error is flat and the statistical phase error resembles a delay. Therefore, a hardware-efficient delay-based digital compensation scheme seems feasible. It uses two coefficients, tuned by a one-step two-tone estimation. It can be implemented using two multipliers and two adders only. The proposed method was applied to cascade-of-poles and ladder active $G_{m}-C$ filters implementing $8.8-\mathrm{MHz}$ seventh-order Butterworth and Chebyshev transfer functions. Simulations showed that the correction improves the image-rejection ratio significantly over the wide bandwidth, e.g., by about $11 \mathrm{~dB}$ for the ladder Butterworth case. In conclusion, the proposed delay-based I/Q compensation offers a fast-estimation (noniterative) and hardware-efficient correction method for telecommunication applications such as 802.11a.

\section{APPENDIX I \\ GAIN AND Phase I/Q IMBALANCE}

An imperfect quadrature signal can be modeled in the phase domain as two rotating phasors with $\omega_{0}$ angular frequency by $(\pi / 2-\phi)$ apart, and with $A_{I}$ and $A_{Q}$ magnitudes, respectively (Fig. 21(a)). The frequency-domain representation of this two-path signal contains a desired component at $\omega_{0}$ and, IMR decibels below it, a leakage (undesired) component at $-\omega_{0}$ [Fig. 21(a)]. The IMR would be infinitely large if the gain imbalance $\gamma \doteq A_{I} / A_{Q}$ was unity and the phase imbalance $\phi$ was zero.
To find out the finite IMR $=\left|A_{p} / A_{n}\right|^{2}$, the pair of $\left\{A_{I}, A_{Q}\right\}$ can be projected to orthogonal axis [Fig. 21(b)]; then, decomposed into a pair of two equal-amplitude and orthogonal phasors [Fig. 21(c)]. It turns out that magnitudes $A_{p}$ and $A_{n}$ give the positive-frequency and negative-frequency components, respectively [Fig. 21(c)].

To derive $A_{p}$ and $A_{n}$ from $A_{I}, A_{Q}$, and $\phi$, simple mathematical steps should be followed. From Fig. 21(a)-(c) results

$$
\left\{\begin{array}{c}
A_{p}-A_{n}=A_{I} \cos \phi \\
A_{p}+A_{n}=A_{Q}+A_{I} \sin \phi .
\end{array}\right.
$$

Solving it for $A_{p}$ and $A_{n}$ gives

$$
\left\{\begin{array}{l}
2 A_{p}=A_{Q}+A_{I} \sin \phi+A_{I} \cos \phi \\
2 A_{n}=A_{Q}+A_{I} \sin \phi-A_{I} \cos \phi
\end{array}\right.
$$

Therefore

$$
\operatorname{IMR}(\gamma, \phi) \doteq\left|\frac{A_{p}}{A_{n}}\right|^{2} \cong\left|\frac{1+\gamma^{2}+2 \gamma \cos \phi}{1+\gamma^{2}-2 \gamma \cos \phi}\right| \approx \frac{\gamma^{2}+\phi^{2}}{4}
$$

Also

$$
\left.\operatorname{IMR}_{\gamma} \doteq \operatorname{IMR}(\gamma, \phi)\right|_{\phi=0}=\left(\frac{1+\gamma}{1-\gamma}\right)^{2}
$$

and

$$
\left.\operatorname{IMR}_{\phi} \doteq \operatorname{IMR}(\gamma, \phi)\right|_{\gamma=1}=\frac{1+\cos \phi}{1-\cos \phi} .
$$

Equation (13) backs up (2), and (14) and (15) were used in Section II-C.

It is interesting to observe the symmetry of $\operatorname{IMR}(\gamma, \phi)$ given in (13): the IMR does not change if $\gamma$ and/or $\phi$ are replaced by $1 / \gamma$ and/or $-\phi$, respectively. This property, once again, confirms that what actually matters is the relative gain and phase mismatch between the $I$ and $Q$ paths and not the absolute values [8].

\section{APPENDIX II \\ ZERO-IF VERSUS LOW-IF FILTERS}

The decomposition of IMR into gain and phase errors (Section II-C) is possible for a pair of real filters since the I/Q imbalance is directly related to the gain and phase mismatch of the two paths. However, in complex filters the image is gradually filtered while passing through the filter; the overall leakage of a complex filter is given by a "leaking-filtering" iterative process [17], [19], [20], which cannot be decomposed into global gain and phase errors. Also, due to this fundamentally different mechanism between complex filters [23]-[27] and a pair of real filters [21], [22], in general, complex filters have better image rejection than a pair of real filters.

Complex filters are usually used in low-IF RX/TX and a pair of real filters are always used in zero-IF RX/TX. Note that complex LPFs were proposed for zero-IF transceivers in [17]. The I/Q leakage mechanism in band-pass and low-pass complex filters is the same. However, band-pass complex filters better reject the image than their low-pass counterpart since they operate at a higher intermediate frequency than dc. Therefore, their I/Q imbalance is lower.

Although complex filters have better image rejection than a pair of real filters, the zero-IF architecture is preferred to a 
low-IF architecture for some applications due to other considerations (such as the strength and location of blockers, etc.) than the filter's IMR alone. Once again, in case of a pair of real filters, the image is "cancelled" at the global output only, since there is no interaction between the $I$-path and $Q$-path internal nodes.

\section{APPENDIX III \\ VARIABLE-DELAY FILTER}

When a signal $x[n]$ is delayed by $\tau=\delta P / \omega_{p}, \tau>0$, then the resulting signal $x_{p}[n]$ can be calculated from $x[n+1]$ and $x[n]$ [Fig. 12(a)]. If the delay is negative, i.e., $\tau \leq 0$, then $x[n-1]$ and $x[n]$ are needed to determine $x_{p}[n]$ [dashed line on Fig. 12(a)]. Therefore

$$
\begin{cases}x_{p}[n]=\left(1-\frac{\delta P}{\omega_{p} T_{s}}\right) x[n]+\frac{\delta P}{\omega_{p} T_{s}} x[n+1], & \text { if } \delta P>0 \\ x_{p}[n]=\left(1-\frac{\delta P}{\omega_{p} T_{s}}\right) x[n]+\frac{-\delta P}{\omega_{p} T_{s}} x[n-1], & \text { if } \delta P \leq 0 .\end{cases}
$$

In the $z$ domain (16) becomes

$$
\begin{cases}\frac{X_{p}(z)}{X(z)}=\left(1-\frac{\delta P}{\omega_{p} T_{s}}\right)+\frac{\delta P}{\omega_{p} T_{s}} z^{+1}, & \text { if } \delta P>0 \\ \frac{X_{p}(z)}{X(z)}=\left(1-\frac{-\delta P}{\omega_{p} T_{s}}\right)+\frac{-\delta P}{\omega_{p} T_{s}} z^{-1}, & \text { if } \delta P \leq 0\end{cases}
$$

which can be rearranged as

$$
\begin{cases}z^{-1} \frac{X_{p}(z)}{X(z)}=\frac{\delta P}{\omega_{p} T_{s}}\left(1-z^{-1}\right)+z^{-1}, & \text { if } \delta P>0 \\ \frac{X_{p}(z)}{X(z)}=\frac{-\delta P}{\omega_{p} T_{s}}\left(z^{-1}-1\right)+1, & \text { if } \delta P \leq 0 .\end{cases}
$$

The one-tap filter, which implements (18), is shown in Fig. 12(b). The gain-correction branch should include a $T_{S}$ delay for $\tau>0$ in order to get synchronized with the phase-correction operations.

\section{ACKNOWLEDGMENT}

The authors gratefully acknowledge the useful discussions with R. Arkiszewski, X.-J. Tao, J. Hammerschmidt, M. Banu, B. Frye, J. Arias and P. Bronner.

\section{REFERENCES}

[1] A. A. Abidi, "Direct-conversion radio transceivers for digital communications," IEEE J. Solid-State Circuits, vol. 30, pp. 1399-1410, Dec. 1995.

[2] B. Razavi, "Design considerations for direct-conversion receivers," IEEE J. Solid-State Circuits, vol. 44, pp. 428-435, June 1997.

[3] — RF Microelectronics. Upper Saddle River, NJ: Prentice-Hall, 1998.

[4] Part 11: Wireless LAN Medium Access Control (MAC) and Physical Layer (PHY) Specifications. High-Speed Physical Layer in the 5-GHz Band, IEEE Standard 802.11a, 1999.

[5] J. K. Cavers and M. W. Liao, "Adaptive compensation for imbalance and offset losses in direct conversion transceivers," IEEE Trans. Veh. Technol., vol. 42, pp. 581-588, Nov. 1993.

[6] J. C. Rudell, J. J. Ou, T. B. Cho, G. Chien, F. Brianti, J. A. Weldon, and P. R. Gray, "A 1.9-GHz wide-band IF double conversion CMOS receiver for cordless telephone applications," IEEE J. Solid-State Circuits, vol. 32, pp. 2071-2088, Dec. 1997.

[7] S. H. Galal, H. F. Ragaie, and M. S. Tawfik, " $R C$ sequence assymmetric polyphase networks for RF integrated transceivers," IEEE Trans. Circuits Syst. II, vol. 47, pp. 18-27, Jan. 2000.

[8] M. Valkama, M. Renfors, and V. Koivunen, "Advanced methods for I/Q imbalance compensation in communication receivers," IEEE Trans. Signal Processing, vol. 49, pp. 2335-2344, Oct. 2001.

[9] J. P. F. Glas, "Digital I/Q imbalance compensation in a low-IF receiver," in Proc. IEEE Global Communications Conf., 1998, pp. 1461-1466.
[10] F. E. Churchill, G. W. Ogar, and B. J. Thompson, "The correction of I and Q errors in a coherent processor," IEEE Trans. Aerosp. Electron. Syst., vol. AES-17, pp. 131-137, Jan. 1981.

[11] K. Pun, J. Franca, and C. Azeredo-Leme, "Wideband digital correction of I and Q mismatch in quadrature radio receivers," in Proc. IEEE Int. Symp. Circuits Systems, 2000, pp. V.661-V.664.

[12] X. J. Tao, "Frequency dependent I/Q calibration," Agere Systems, Allentown, PA, Tech. Memor., 2001.

[13] L. Der and B. Razavi, "A 2-GHz CMOS image-reject receiver with LMS calibration," IEEE J. Solid-State Circuits, vol. 38, pp. 167-175, Feb. 2003.

[14] L. Yu and W. M. Snelgrove, "A novel adaptive mismatch cancellation system for quadrature IF radio receivers," IEEE Trans. Circuits Syst. II, vol. 46, pp. 789-801, June 1999.

[15] K. Pun, J. Franca, and C. Azeredo-Leme, "The correction of frequencydependent I/Q mismatches in quadrature receivers by adaptive signal separation," in Proc. Int. Conf. Application Specified Integrated Circuit, 2001, pp. 424-427.

[16] G. H. Burgin, "Method and apparatus for determination of predistortion parameters for a quadrature modulator," U.S. Patent 6298 096, Oct. 2, 2001.

[17] P. Kiss, V. Prodanov, and J. Glas, "Complex low-pass filters," Int. J. Anal. Integr. Circuits Signal Processing, vol. 35, no. 1, pp. 9-23, 2003.

[18] R. H. Allen, "Complex analog filter obtained from shifted lowpass prototypes," Master's thesis, Dept. Elect. Comput. Eng., Univ. of Toronto,, Toronto, ON, Canada, 1985.

[19] S. A. Jantzi, K. W. Martin, and A. S. Sedra, "Quadrature bandpass delta-sigma modulation for digital radio," IEEE J. Solid-State Circuits, vol. 32, pp. 1935-1950, Dec. 1997.

[20] S. A. Jantzi, "Quadrature bandpass delta-sigma modulation for digital radio," Ph.D. dissertation, Dept. Elect. Comput. Eng., Univ. of Toronto, Toronto, Canada, 1997.

[21] A. I. Zverev, Handbook of Filter Synthesis. New York: Wiley, 1967.

[22] R. Schaumann and M. E. van Valkenburg, Design of Analog Filters. New York: Oxford Univ. Press, 2001.

[23] D. Gabor, "Theory of communication," J. Inst. Elect. Eng., pt. III, vol. 93, pp. 429-457, 1946.

[24] M. J. Gingell, "A symmetrical polyphase network," U.S. Patent 3559042 , June 7, 1968.

[25] — , "The synthesis and application of polyphase networks with sequence asymmetric properties," Ph.D. dissertation, Faculty of Engineering, Univ. of London, London, U.K., 1975.

[26] G. R. Lang and P. O. Brackett, "Complex analogue filters," in Proc. Eur. Conf. Circuit Theory Design, 1981, pp. 412-419.

[27] W. M. Snelgrove and A. S. Sedra, "State-space synthesis of complex analog filters," in Proc. Eur. Conf. Circuit Theory Design, 1981, pp. $420-424$

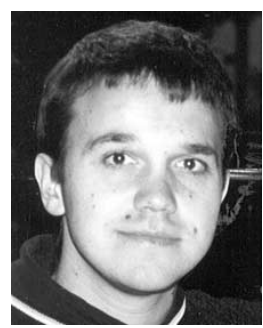

Péter Kiss (S'99-M'00) received the Engineer's, M.S., and Ph.D. degrees, from the Technical University of Timişoara, Timişoara, Romania, in 1994, 1995 and 2000, respectively, all in electrical engineering.

From 1998 to 2000, he was a Research Scholar at Oregon State University, Corvallis, working on correction techniques for fast and accurate delta-sigma converters. Since 2001, he has been with Agere Systems (formerly part of Bell Laboratories, Lucent Technologies), Murray Hill, NJ, dealing with data converters and analog filters for wireless systems. His past work involved adaptive fuzzy systems and image processing.

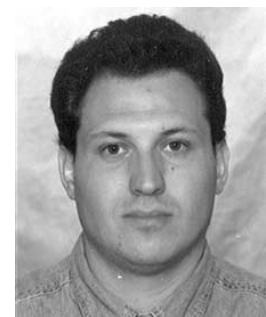

Vladimir Prodanov (S'94-M'97) received the B.S. degree from the Technical University, Sofia, Bulgaria, and the M.S. and Ph.D. degrees from the State University of New York, Stony Brook, in 1991, 1995, and 1997, respectively.

$\mathrm{He}$ is an Analog Circuit Designer in the Communication Circuits Department, Agere Systems (formerly part of Bell Laboratories, Lucent Technologies), Allentown, PA. He is also an Adjunct Professor at Columbia University, New York City, where he teaches analog and RF circuit design. 